\title{
The Tunable Electronic, Magnetic and Curie Temperature by Co Doping $\mathrm{Fe}_{2-x} \mathrm{Co}_{x} \mathrm{MnAl}$ Alloys
}

\author{
Xiao-Ping Wei ${ }^{a, *}$, Sheng-Ming Ma ${ }^{a}$, Xiao-Wei Sun ${ }^{a}$, Peifeng GaO $^{b}$ \\ AND YA-LING ZHANG ${ }^{c}$
}

${ }^{a}$ The School of Mathematics and Physics, Lanzhou Jiaotong University, Lanzhou 730070, P.R. China

${ }^{b}$ Key Laboratory of Mechanics on Western Disaster and Environment, MoE,

College of Civil Engineering and Mechanics, Lanzhou University, Lanzhou 730000, P.R. China

${ }^{c}$ Institute of Modern Physics, Chinese Academy of Sciences, Lanzhou 730000, P.R. China

(Received May 10, 2019; revised version July 22, 2019; in final form August 5, 2019)

Using the spin-polarized relativistic Korringa-Kohn-Rostoker method, we study the electronic, magnetic, and Curie temperature of $\mathrm{Fe}_{2-x} \mathrm{Co}_{x} \mathrm{MnAl}$ alloys. Results indicate that the $\mathrm{Fe}_{2-x} \mathrm{Co}_{x} \mathrm{MnAl}$ alloys preserve the ferromagnetic metallic nature, and the spin polarization is close to $80 \%$ when the Co concentration attains to $30 \%$. The calculated total magnetic moment increases linearly as the increase of Co doping concentration, only small changes are observed for $\mathrm{Fe}(\mathrm{A}), \mathrm{Mn}(\mathrm{B})$, and $\mathrm{Fe}(\mathrm{C})$ atomic magnetic moments, while for the $\mathrm{Al}$ atom, the magnetic moment always nearly approach to zero. The Heisenberg exchange calculations imply that the $\mathrm{Co}(\mathrm{A})-\mathrm{Mn}(\mathrm{B})$ exchange plays a leading role in interactions, in contrast to the $\mathrm{Mn}(\mathrm{B})-\mathrm{Mn}(\mathrm{B})$ exchange in pure $\mathrm{Fe}_{2} \mathrm{MnAl}$. We further calculate the sum of exchange coupling parameters between the constituents, and evaluate the Curie temperature of $\mathrm{Fe}_{2-x} \mathrm{Co}_{x} \mathrm{MnAl}$ alloys. It is found that the $\mathrm{Mn}(\mathrm{B})-\mathrm{Mn}(\mathrm{B})$ and $\mathrm{Fe} / \mathrm{Co}(\mathrm{A})-\mathrm{Mn}(\mathrm{B})$ coupling parameters increase evidently with the increase of Co content, and then leading to the Curie temperature above room temperature when the Co doping concentration is over $60 \%$, the change is also in good consistence with calculated total magnetic moment.

DOI: 10.12693/APhysPolA.136.520

PACS/topics: 71.20.Lp, 74.20.Pq, 51.60.+a, 71.70.Gm

\section{Introduction}

The Heusler alloys, which are firstly discovered by Heusler from the $\mathrm{Cu}_{2} \mathrm{MnAl}$ behaving like ferromagnet, although its constituent elements itself are not magnetic [1], have attracted wide attention because of their potential applications in cutting edge technology in spintronics [2-7]. Particularly, the Heusler alloys with high Curie temperature and high spin polarization are more favorable in realistic applications. However, some cheaper and more promising Heusler alloys do not show these interesting physical properties. In this case, we usually tune their electron density to achieve the physical properties of interest such as high Curie temperature and high spin polarization through an appropriate choice of substitutional doping meanwhile retaining their essential structures [8-11].

Recently, $\mathrm{Fe}_{2} \mathrm{Mn}$-based Heusler alloys have drawn widespread attention from both theoretical and experimental aspects [12-17]. Especially, $\mathrm{Fe}_{2} \mathrm{MnZ}(\mathrm{Z}=\mathrm{Sn}, \mathrm{Al}, \mathrm{Si})$ alloys have been synthesized in experiment [18-20]. Among these alloys, $\mathrm{Fe}_{2} \mathrm{MnAl}$ is promising material since it is made up of common elements, which makes it possible to be widely used in various spintronics devices. However, a lower Curie

\footnotetext{
*corresponding author; e-mail: weixp2008@gmail.com
}

temperature and spin polarization limit its application in room temperature spintronics devices $[16,17]$.

In this work, we will take $\mathrm{Fe}_{2} \mathrm{MnAl}$ as the object of study, and tune its electron density by doping $\mathrm{Co}$ at $\mathrm{Fe}(\mathrm{A})$ site in the series of $\mathrm{Fe}_{2-x} \mathrm{Co}_{x} \mathrm{MnAl}$ alloys in order to obtain interesting physical properties such as high Curie temperature and high spin polarization, etc. This research can broaden the application scope of $\mathrm{Fe}_{2} \mathrm{MnAl}$. We also can understand the inherent physical mechanism resulting in the changes of the Curie temperature and spin polarization of $\mathrm{Fe}_{2} \mathrm{MnAl}$, and then providing a feasibility for the improvement of the physical properties of such alloys.

More specifically, the paper is organized as follows. Calculation details are given in Sect. 2, followed by the results and discussion in Sect. 3. Finally, we present a conclusion in Sect. 4.

\section{Calculation details}

In current calculations, we use the coherence potential approximation within spin-polarized scalar relativistic Korringa-Kohn-Rostoker package Munich SPRKKR [21]. The exchange-correlation potential was modeled by using the Perdew-Burke-Ernzerhof (PBE) parametrization in scheme of the generalized gradient approximations (GGA) [22]. An angular momentum cut-off of $l_{\max }=3$ and $22 \times 22 \times 22 \mathrm{k}$-point mesh in the irreducible wedge of the Brillouin zone are used in calculations. 
By using the exchange coupling parameters $J_{i j}$, we calculate the Curie temperature with mean-field approximation (MFA). In the classical Heisenberg model, the Hamiltonian of a spin system is given by

$$
H=-\sum_{i, j} e_{i} e_{j} J_{i j},
$$

with the Heisenberg pair exchange coupling parameters $J_{i j}$, and unit vectors $e_{i}\left(e_{j}\right)$ pointing in the direction of the magnetic moment on site $i(j)$. The Heisenberg exchange coupling parameter $J_{i j}$ were calculated via the Liechtenstein-Katsnelson-Antropov-Gubanov (LKAG) formalism as [23]:

$$
J_{i j}=\frac{1}{\pi} \int_{-\infty}^{\varepsilon_{F}} \operatorname{Im}\left[\operatorname{Tr}\left(\Delta_{\mathrm{i}} \tau_{\mathrm{ij}}^{\uparrow} \Delta_{\mathrm{j}} \tau_{\mathrm{ji}}^{\downarrow}\right)\right] \mathrm{d} \varepsilon,
$$

where $\Delta_{i}=t_{i, \uparrow}^{-1}-t_{i, \downarrow}^{-1}$ is the spin-resolved difference of single-site scattering matrix $t_{i}$ at site $i$, and $\tau_{i j}$ is the scattering path operator, describing the propagation of the electrons between two sites $i$ and $j$. The Fermi energy is denoted by $\varepsilon_{F}$. According to $J_{i j}$, the Curie temperature can be estimated within the MFA. More detailed calculations on the Curie temperature can be seen in our previous works $[24,25]$. In MFA, we construct a $4 \times 4$ matrix to obtain the Curie temperature, and adopt pair exchange coupling parameters up to $r_{\max }=4.0 \mathrm{a}$.u. It needs to be stressed that the atomic $J_{i j}$ of A site with other atoms equals to the sum of $J_{i j}^{F e}$ and $J_{i j}^{C o}$.

\section{Results and discussion}

\subsection{Equilibrium states and lattice parameters}

Initially, we build structural model of a series of $\mathrm{Fe}_{2-x} \mathrm{Co}_{x} \mathrm{MnAl}$ alloys based on $\mathrm{Fe}_{2} \mathrm{MnAl}$ which has been confirmed in experiment crystallizing in the $L 2_{1}$ structure [13]. In the structure, the Fe atoms occupy $\mathrm{A}(0,0,0)$ and $\mathrm{C}\left(\frac{1}{2}, \frac{1}{2}, \frac{1}{2}\right)$ Wyckoff position, and the $\mathrm{Mn}$ and $\mathrm{Al}$ atoms are located on $\mathrm{B}\left(\frac{1}{4}, \frac{1}{4}, \frac{1}{4}\right)$ and $\mathrm{D}\left(\frac{3}{4}, \frac{3}{4}, \frac{3}{4}\right)$ positions, respectively. Among these positions, the Fe atom located at A site is partially substituted by Co element. In order to obtain the equilibrium states of $\mathrm{Fe}_{2-x} \mathrm{Co}_{x} \mathrm{MnAl}$ alloys at different Co doping concentration, we fit the volume-energy date by using the Murnaghan equation [26]. The fitted equilibrium parameters are tabulated in Table I. From Table I, we notice that the total energies of doped systems are much lower than pure $\mathrm{Fe}_{2} \mathrm{MnAl}$, indicating that they are possible to be synthesized in experiment. In addition, other available theoretical and experimental values are also given for comparison, it is obvious that our results are well consistent with others $[8,14,17,27]$.

\subsection{Density of states and magnetic moments}

Based on the equilibrium states, we calculate the density of states (DOS) of $\mathrm{Fe}_{2-x} \mathrm{Co}_{x} \mathrm{MnAl}$ alloys, and show them in Fig. 1. From these calculated DOS, we notice
TABLE I

The calculated lattice parameter ( $a$ in $\AA$ ), bulk modulus $\left(B_{0}\right.$ in $\left.\mathrm{GPa}\right)$, total energy ( $E_{\mathrm{tot}}$ in Ry) and Curie temperature $\left(T_{\mathrm{C}}\right.$ in $\left.\mathrm{K}\right)$ for $\mathrm{Fe}_{2-x} \mathrm{Co}_{x} \mathrm{MnAl}$ alloys.

\begin{tabular}{c|c|c|c|c|c|c}
\hline \hline$x$ & $a_{\text {calc }}$ & $a_{\text {calc }}$ & $a_{\exp }$ & $B_{0}$ & $E_{\text {tot }}$ & $T_{\mathrm{C}}$ \\
\hline 0.0 & 5.618 & $5.680^{a}\left(5.62^{c}\right)$ & $5.816^{b}$ & 187.4 & -7894.32 & 4.24 \\
0.1 & 5.613 & - & - & 166.3 & -7918.45 & 109.32 \\
0.2 & 5.618 & - & - & 182.7 & -7942.59 & 155.88 \\
0.3 & 5.628 & - & - & 168.7 & -7966.73 & 205.91 \\
0.4 & 5.621 & - & - & 207.0 & -7990.86 & 245.72 \\
0.5 & 5.626 & $5.63^{c}$ & - & 197.3 & -8015.00 & 281.58 \\
0.6 & 5.632 & - & - & 195.4 & -8039.13 & 313.02 \\
0.7 & 5.663 & - & - & 218.3 & -8063.27 & 341.75 \\
0.8 & 5.665 & - & - & 213.2 & -8087.41 & 367.24 \\
0.9 & 5.634 & - & - & 168.9 & -8111.55 & 392.00 \\
1.0 & 5.669 & $5.69^{c}\left(5.692^{d}\right)$ & $5.786^{d}$ & 305.8 & -8135.69 & 384.65 \\
\hline${ }^{a}$ Ref. [14], ${ }^{b}$ Ref. [17], ${ }^{c}$ Ref. [8], ${ }^{d}$ Ref. $[27]$
\end{tabular}

that the transition metal $3 d$ states offer a main contribution to total DOS for a series of $\mathrm{Fe}_{2-x} \mathrm{Co}_{x} \mathrm{MnAl}$ alloys, and the $d-d$ orbital hybridization plays a main role around the Fermi level. In addition, the spin-up states occupy majority-spin states compared with spindown states, and then the $\mathrm{Fe}_{2-x} \mathrm{Co}_{x} \mathrm{MnAl}$ alloys are ferromagnets, and indicates the metallic behaviour since the Fermi level goes through the both spin-up and spindown DOS. The Fermi level is pushed to pseudogap of spin-up states when Co dopant is in a $0.1-0.4$ range, and deviate from the pseudogap with the further increase of Co doping concentration. It is due to the decrease of exchange splitting of $d-d$ hybridization states. It is also shown by spin polarization in Fig. 2, that the spin polarization is increasing when Co content increases within $x \leq 0.4$. Particularly, the spin polarization nearly closes to $80 \%$ when $x=0.4$. In addition, we also note that the polarization is always above $40 \%$ at different Co doping concentration. With more details, from the calculated total magnetic moments as shown in Table II, we notice that the total magnetic moments of $\mathrm{Fe}_{2-x} \mathrm{Co}_{x} \mathrm{MnAl}$ alloys nearly obey the Slater-Pauling rule $M_{t}=Z_{t}-24[28,29]$, where $M_{t}$ is the calculated total magnetic moment, and $Z_{t}$ is the valence electrons number per formula unit. Small deviation from integer is ascribed to adopted KKR method. $\mathrm{Fe}_{2-x} \mathrm{Co}_{x} \mathrm{MnAl}$ alloys are not half-metals due to the conduction electrons at the Fermi level unpolarized fully. It is also confirmed by calculated DOS and spin polarization in Figs. 1 and 2 .

In addition, we also notice that the $\mathrm{Mn}(\mathrm{B})$ atom offers a main contribution to total magnetic moment. The dependences of magnetic moments on Co doping concentration are presented in Fig. 3. It can be seen that total magnetic moment increases almost linearly with the increase of Co doping content, the change is attributed to the increase of the $\mathrm{Mn}(\mathrm{B})$ and $\mathrm{Co}(\mathrm{A})$ atomic moments. In contrast, small change is observed for the magnetic moments of Fe atoms. For the $\mathrm{Al}$ atom, the magnetic moment nearly becomes zero because of the $\mathrm{Al}$ unpolarized atom. 

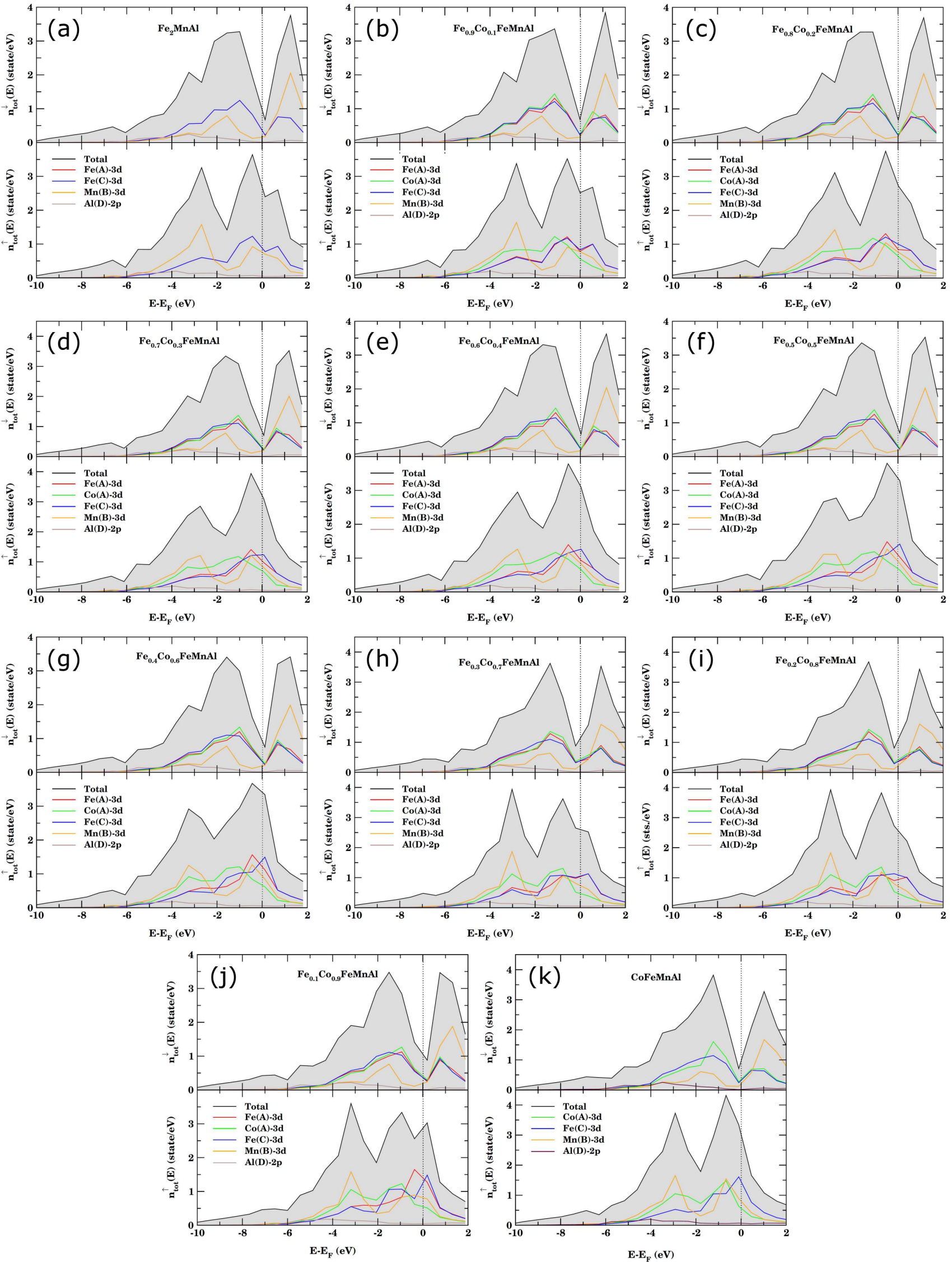

Fig. 1. Total and resolve density of states for a series of $\mathrm{Fe}_{2-x} \mathrm{Co}_{x} \mathrm{MnAl}$ alloys. 
Total and atomic magnetic moments (in $\mu_{\mathrm{B}}$ units) of $\mathrm{Fe}_{2-x} \mathrm{Co}_{x} \mathrm{MnAl}$ alloys are tabulated and compared

TABLE II with available results by reference.

\begin{tabular}{c|c|c|c|c|c|c|c}
\hline \hline$x$ & $M_{\text {calc }}$ & $M_{\text {calc }}$ & $\mathrm{Fe}(\mathrm{A})$ & $\mathrm{Co}(\mathrm{A})$ & $\mathrm{Fe}(\mathrm{C})$ & $\mathrm{Mn}(\mathrm{B})$ & $\mathrm{Al}(\mathrm{D})$ \\
\hline 0.0 & 2.00 & $2.01^{a}$ & $-0.20\left(-0.27^{a}\right)$ & - & $-0.20\left(-0.27^{a}\right)$ & $2.44\left(2.29^{a}\right)$ & $-0.04\left(-0.01^{a}\right)$ \\
0.1 & 2.10 & - & -0.12 & 0.59 & -0.24 & 2.44 & -0.04 \\
0.2 & 2.20 & - & -0.10 & 0.65 & -0.28 & 2.47 & -0.04 \\
0.3 & 2.30 & - & -0.09 & 0.70 & -0.30 & 2.50 & -0.05 \\
0.4 & 2.40 & - & -0.09 & 0.73 & -0.29 & 2.51 & -0.05 \\
0.5 & 2.51 & $2.50^{a}$ & -0.09 & 0.75 & -0.30 & 2.53 & -0.06 \\
0.6 & 2.61 & - & -0.09 & 0.76 & -0.30 & 2.55 & -0.06 \\
0.7 & 2.71 & - & -0.11 & 0.77 & -0.35 & 2.62 & -0.07 \\
0.8 & 2.80 & - & -0.11 & 0.78 & -0.35 & 2.63 & -0.07 \\
0.9 & 2.90 & - & -0.10 & 0.79 & -0.31 & 2.59 & -0.08 \\
1.0 & 2.98 & $3.00^{a}$ & - & $0.79\left(0.81^{a}\right)$ & $-0.40\left(-0.14^{a}\right)$ & $2.67\left(2.44^{a}\right)$ & $-0.08\left(-0.04^{a}\right)$ \\
\hline
\end{tabular}

${ }^{a}$ Ref. [8]

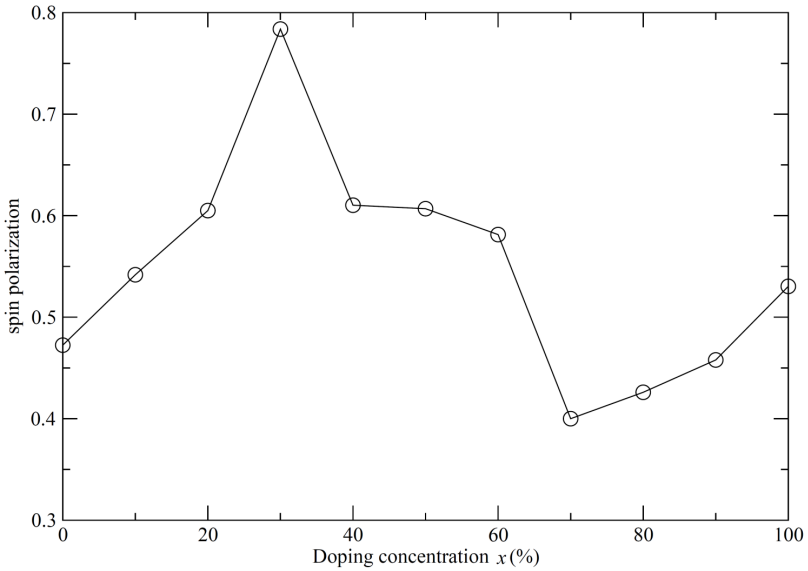

Fig. 2. Spin polarization at different Co doping concentration.

\subsection{Exchange interactions and Curie temperatures}

In Fig. 4, we show the calculated Heisenberg exchange coupling parameters $J_{i j}$. It is evident that the exchange interactions are tightly restricted to clusters of radius $r \leq 2.0 \mathrm{a}$. Specifically, the intra-sublattice $\mathrm{Mn}(\mathrm{B})-\mathrm{Mn}(\mathrm{B})$ interaction provides significant contribution in overall interactions in $\mathrm{Fe}_{2} \mathrm{MnAl}$, and the interaction between the first nearest neighbor and second nearest neighbor is ferromagnetic, and the third nearest neighbor is antiferromagnetic interaction. The intersublattices $\mathrm{Fe}(\mathrm{A})-\mathrm{Mn}(\mathrm{B})$ and $\mathrm{Fe}(\mathrm{C})-\mathrm{Mn}(\mathrm{B})$ as well as intra-sublattice $\mathrm{Fe}(\mathrm{A})-\mathrm{Fe}(\mathrm{C})$ exchanges between the first and second nearest neighbor are ferromagnetic, and the sum of $J_{i j}$ is rather small, approaching nearly zero. For the $\mathrm{Fe}_{0.9} \mathrm{Co}_{0.1} \mathrm{FeMnAl}$ alloy, the inter-sublattice $\mathrm{Co}(\mathrm{A})-\mathrm{Mn}(\mathrm{B})$ exchange plays a leading role in all interactions, followed by the intra-sublattice $\mathrm{Mn}(\mathrm{B})-\mathrm{Mn}(\mathrm{B})$. The inter-sublattice $\mathrm{Co}(\mathrm{A})-\mathrm{Mn}(\mathrm{B})$ exchange between the first nearest neighbor and second nearest neighbor is antiferromagnetic interaction. In contrast, it is ferromagnetic interaction for the intra-sublattice $\mathrm{Mn}(\mathrm{B})-\mathrm{Mn}(\mathrm{B})$

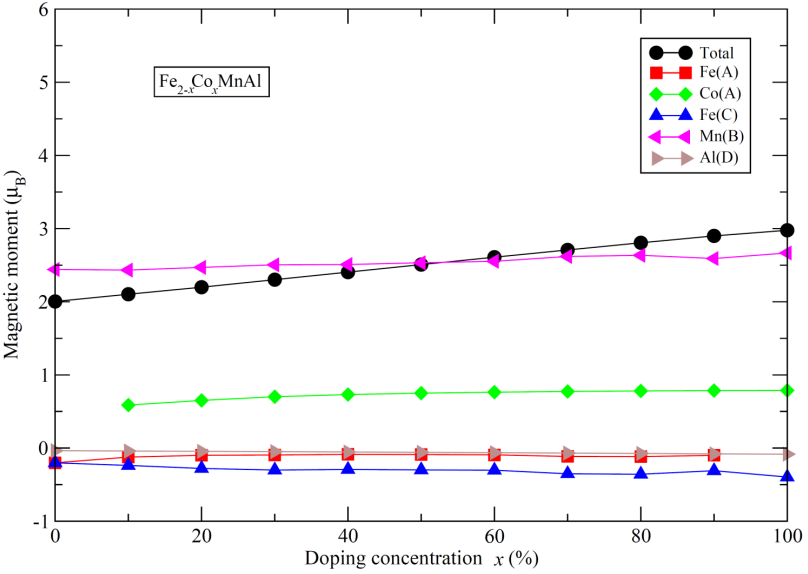

Fig. 3. Total and atomic magnetic moments on different Co doping concentration for a series of $\mathrm{Fe}_{2-x} \mathrm{Co}_{x} \mathrm{MnAl}$ alloys.

exchange. In $\mathrm{Fe}_{0.8} \mathrm{Co}_{0.2} \mathrm{FeMnAl}$ alloy, the situation is the same as in the case of $\mathrm{Fe}_{0.9} \mathrm{Co}_{0.1} \mathrm{FeMnAl}$, the difference is that the ferromagnetic interaction is stronger. In the $\mathrm{Fe}_{0.7} \mathrm{Co}_{0.3} \mathrm{FeMnAl}$ alloy, the intersublattice $\mathrm{Co}(\mathrm{A})-\mathrm{Mn}(\mathrm{B})$ and $\mathrm{Fe}(\mathrm{C})-\mathrm{Mn}(\mathrm{B})$ interactions become further stronger between the first nearest neighbor and second nearest neighbor, and the intra-sublattice $\mathrm{Mn}(\mathrm{B})-\mathrm{Mn}(\mathrm{B})$ exchange between the first and second nearest neighbor are smaller. Further, the ferromagnetic exchanges of inter-sublattices $\mathrm{Co}(\mathrm{A})-\mathrm{Mn}(\mathrm{B})$ and $\mathrm{Fe}(\mathrm{C})-\mathrm{Mn}(\mathrm{B})$ further increase with the increase in Co doping concentration. It needs to be emphasized that the interactions with $\mathrm{Al}$ are omitted, because it is close to zero for all distances. In the $\mathrm{Fe}_{1-x} \mathrm{Co}_{x} \mathrm{FeMnAl}$ alloys, the $J_{i j}$ shows a oscillatory behavior with increase in interatomic distances $r$, implying a RKKY exchange [30]. Finally, the behavior disappears with increase in interatomic distance. In addition, we also offer the sum of $J_{i j}$ in Fig. 5, where it can be seen that the sum of intra-sublattices 

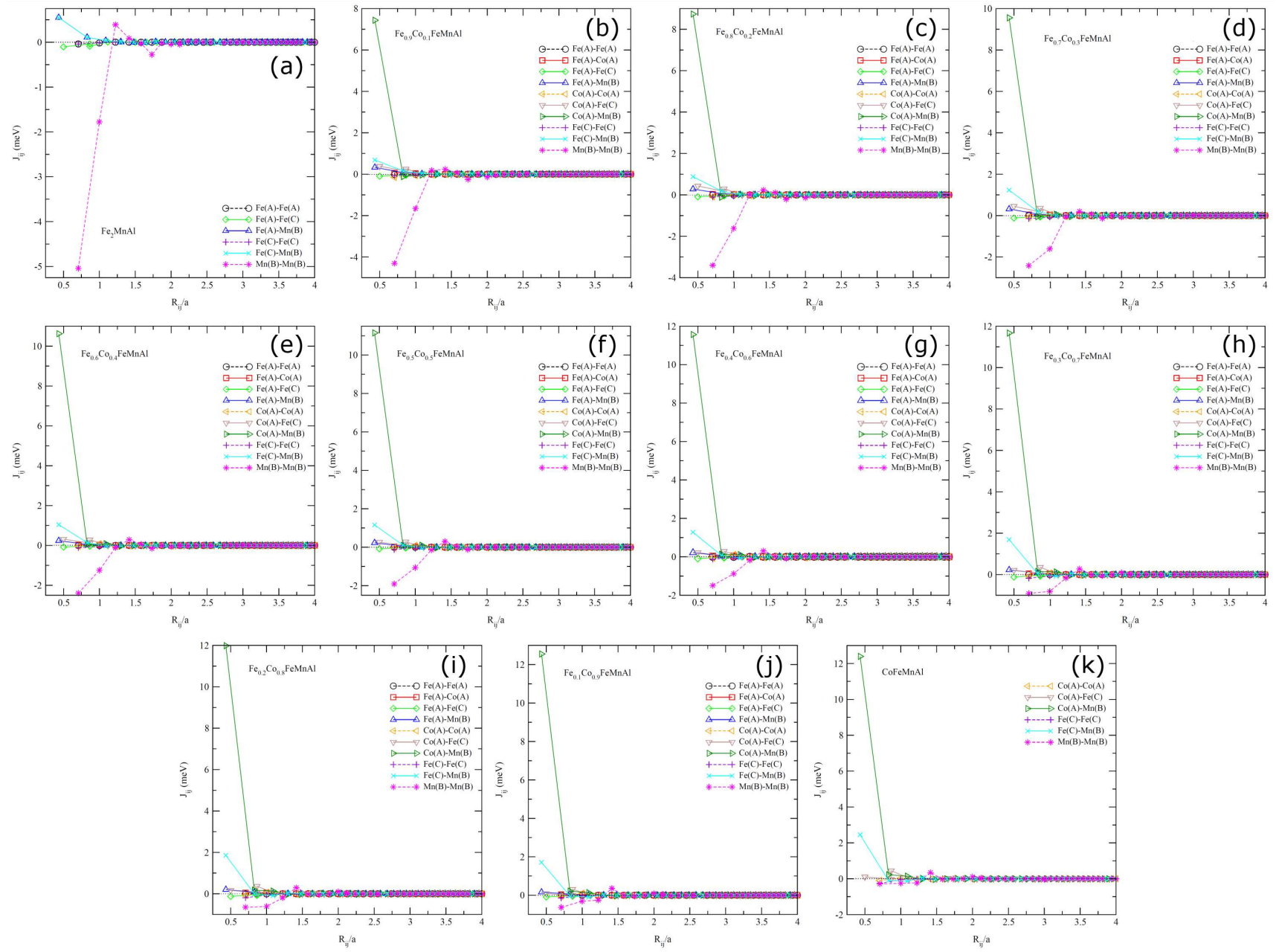

Fig. 4. Heisenberg exchange coupling parameters $J_{i j}$ for a series $\mathrm{Fe}_{2-x} \mathrm{Co}_{x} \mathrm{MnAl}$ alloys as a function of the interatomic distance $r$.

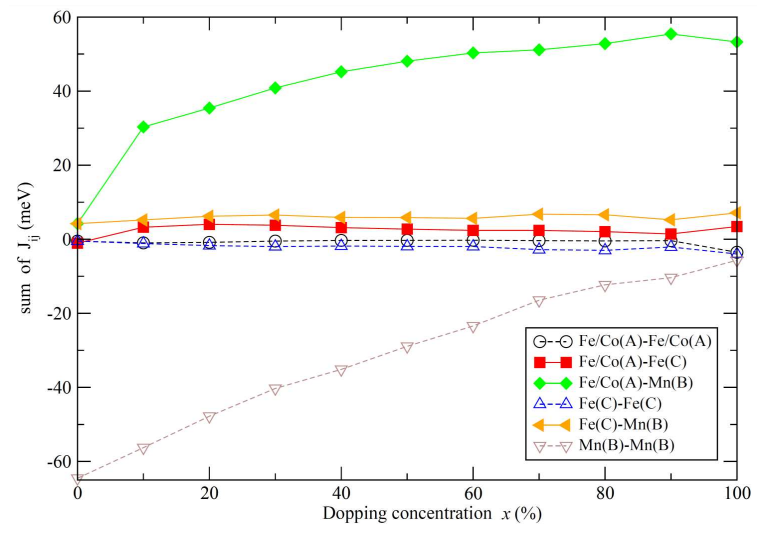

Fig. 5. Sum of exchange interaction $J_{i j}$ between different sites for a series of $\mathrm{Fe}_{2-x} \mathrm{Co}_{x} \mathrm{MnAl}$ alloys.

$\mathrm{Fe}(\mathrm{C})-\mathrm{Fe}(\mathrm{C})$ and $\mathrm{Fe} / \mathrm{Co}(\mathrm{A})-\mathrm{Fe} / \mathrm{Co}(\mathrm{A})$ exchange is nearly zero. For the inter-sublattices $\mathrm{Fe} / \mathrm{Co}(\mathrm{A})-\mathrm{Fe}(\mathrm{C})$ and $\mathrm{Fe}(\mathrm{C})-\mathrm{Mn}(\mathrm{B})$ exchange, the sum of coupling parameters are about $5 \mathrm{meV}$ and $8 \mathrm{meV}$, respectively. For the intersublattice $\mathrm{Fe} / \mathrm{Co}(\mathrm{A})-\mathrm{Mn}(\mathrm{B})$ exchange, the sum of $J_{i j}$ is gradually increasing as the increase of Co doping concentration, and approaching to $55 \mathrm{meV}$ at $x=0.8$. For the intra-sublattice $\mathrm{Mn}(\mathrm{B})-\mathrm{Mn}(\mathrm{B})$ exchange, the sum of $J_{i j}$ hardly increases linearly, and the value is largest at $x=0$, up to $65 \mathrm{meV}$. Actually, a negative $J_{i j}$ implies that the interaction acts against the ferromagnetic order on this lattice and reduces the Curie temperature. By contrast, a positive $J_{i j}$ can improve the Curie temperature.

Based on the exchange coupling parameters described above, we calculate the Curie temperature for different Co doping concentration by the MFA, and show them in Table I. In Figure 6, we also show the calculated Curie temperature dependence in the Co doping concentration. It is found that the Curie temperature is increasing with the increase of Co doping concentration. The Curie temperature up to $313.02 \mathrm{~K}$ when the Co doping concentration is up to $x=0.6$, and further increase with the increase of Co doping concentration, finally reaching $392.00 \mathrm{~K}$ when $x=0.9$, which is evidently above room temperature, indicating their potential magnetic applications. By comparing with Fig. 3, we 


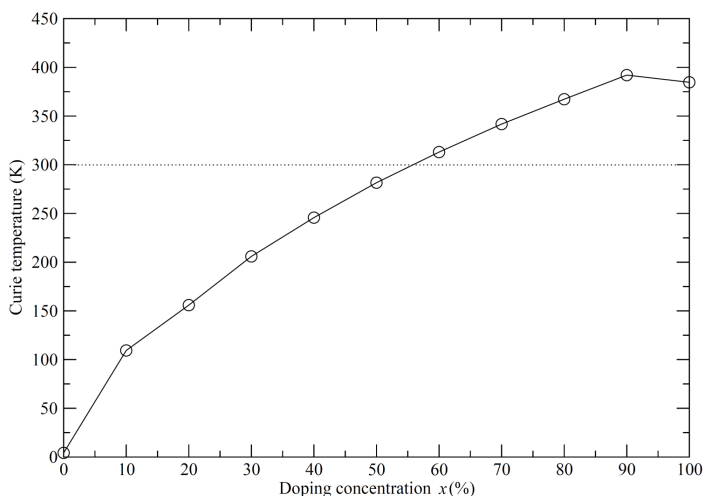

Fig. 6. Curie temperature as a function of different Co doping concentration. Note that the dotted line represents the room temperature.

observe that the Curie temperatures of $\mathrm{Fe}_{2-x} \mathrm{Co}_{x} \mathrm{MnAl}$ alloys are roughly proportional to the total magnetic moment.

\section{Summary and conclusion}

We have performed the electronic, magnetic, and Curie temperature calculations on the $\mathrm{Fe}_{2-x} \mathrm{Co}_{x} \mathrm{MnAl}$ alloys with $L 2_{1}$ structure by using the SPRKKR method. It is found that the $\mathrm{Fe}_{2-x} \mathrm{Co}_{x} \mathrm{MnAl}$ alloys are ferromagnets and show metallic behavior. However, the calculated total magnetic moments nearly obey the Slater-Pauling rule. The $\mathrm{Mn}(\mathrm{B})$ atomic magnetic moment is increasing with the increase of Co doping concentration. Only small change is observed for the moments of $\mathrm{Fe}$ atoms, the magnetic moment of unpolarized $\mathrm{Al}$ atom is nearly zero. From the calculated exchange interactions, it is found that the inter-sublattice $\mathrm{Fe} / \mathrm{Co}(\mathrm{A})-\mathrm{Mn}(\mathrm{B})$ exchange plays a leading role in all interactions, and finally determines the Curie temperature. Further, we estimate the Curie temperatures of different Co doping concentration by using MFA. Results indicate that the Curie temperature is above room temperature when the Co doping concentration is over $60 \%$. More noticeably, the Curie temperatures of $\mathrm{Fe}_{2-x} \mathrm{Co}_{x} \mathrm{MnAl}$ alloys are roughly proportional to the total magnetic moment. We hope that the study can provide some valuable hints for further performance improvement of such alloys.

\section{Acknowledgments}

The project is supported by National Natural Science Foundation of China (No. 11864021). The work is also supported by Foundation of A Hundred Youth Talents Training Program of Lanzhou Jiaotong University. Peifeng Gao would like to acknowledge the supports by the China Postdoctoral Science Foundation (No. 2018M633604) and the Fundamental Research Funds for the Central Universities (No. lzujbky-2018-it01).

\section{References}

[1] F. Heulser, Verhandl. Deut. Physik. Ges. 5, 219 (1903).

[2] L. Bainsla, K.G. Suresh, Appl. Phys. Rev. 3, 031101 (2016).

[3] B. Dieny, V.S. Speriosu, S.S.P. Parkin, B.A. Gurney, D.R. Wilhoit, D. Mauri, Phys. Rev. B 43, 1297 (1991).

[4] S.A. Wolf, D.D. Awschalom, R.A. Buhrman, J.M. Daughton, S.V. Molnar, M.L. Roukes, A.Y. Chtchelkanova, D.M. Treger, Science 294, 1488 (2001)

[5] S.S.P. Parkin, M. Hayashi, L. Thomas, Science 320, 190 (2008).

[6] O. Gutfleisch, M.A. Willard, E. Brück, C.H. Chen, S.G. Sankar, J.P. Liu, Adv. Mater. 23, 821 (2011).

[7] T. Graf, C. Felser, S.S.P. Parkin, Prog. Solid State Chem. 39, 1 (2011).

[8] V.K. Jain, N. Lakshmi, R. Jain, V. Jain, A.R. Chandra, K. Venugopalan, J. Mater. Sci. 52, 6800 (2017).

[9] X.F. Zhu, L. Wang, Eur. Phys. J. B 90, 64 (2017).

[10] R. Jain, N. Lakshmi, V. K. Jain, A. R. Chandra, AIP Conf. Proc. 1942, 090011 (2018.

[11] I. Galanakis, K. Özdoğan, B. Aktaş, Appl. Phys. Lett. 89, 042502 (2006).

[12] Y. Jirásková, J. Buršík, D. Janičkovič, O. Životský, Materials 12, 710 (2019).

[13] N.I. Kourov, V.V. Marchenkov, K.A. Belozerova, H.W. Weber, J. Exp. Theor. Phys. 118, 426 (2014).

[14] M. Belkhouane, S. Amari, A. Yakoubi, A. Tadjer, S. Méçbih, G. Murtaza, S. Bin Omran, R. Khenata, J. Magn. Magn. Mater. 377, 211 (2015).

[15] V.K. Jain, N. Lakshmi, R. Jain, A.R. Chandra, J. Supercond. Nov. Magn. 32, 739 (2018).

[16] F. Dahmane, Y. Mogulkoc, B. Doumi, A. Tadjer, R. Khenata, S.B. Omran, D.P. Rai, G. Murtaza, D. Varshney, J. Magn. Magn. Mater. 407, 167 (2016).

[17] Z.H. Liu, X.Q. Ma, F.B. Meng, G.H. Wu, J. Alloys Comp. 509, 3219 (2011).

[18] V.K. Jain, N. Lakshmi, V. Jain, A.K. Sijo, K. Venugopalan, AIP Conf. Proc. 1665, 130032 (2015).

[19] A. Vinesh, H. Bhargava, N. Lakshmi, K. Venugopalan, J. Appl. Phys. 105, 07A309 (2009).

[20] N.I. Kourov, V.V. Marchenkov, A.V. Korolev, L.A. Stashkova, S.M. Emel'yanova, H.W. Weber, Phys. Solid State 57, 700 (2015).

[21] H. Ebert, D. Ködderitzsch, J. Minár, Rep. Prog. Phys. 74, 096501 (2011).

[22] J.P. Perdew, K. Burke, M. Ernzerhof, Phys. Rev. Lett. 77, 3865 (1996).

[23] A.I. Liechtenstein, M.I. Katsnelson, V.P. Antropov, V.A. Gubanov, J. Magn. Magn. Mater. 67, 65 (1987).

[24] X.P. Wei, Y.L. Zhang, X.W. Sun, T. Song, P. Guo, Y. Gao, J.L. Zhang, X.F. Zhu, J.B. Deng, J. Alloys Comp. 694, 1254 (2017).

[25] X.P. Wei, X.W. Sun, T. Song, J.H. Tian, P. Guo, J. Supercond. Nov. Magn. 31, 2797 (2018). 
[26] F.D. Murnaghan, Am. J. Math. 59, 235 (1937).

[27] V. Alijani, S. Ouardi, G.H. Fecher, J. Winterlik, S.S. Naghavi, X. Kozina, G. Stryganyuk, C. Felser, Phys. Rev. B 84, 224416 (2011).

[28] S. Skaftouros, K. Özdoğan, E. Şaşioğlu, I. Galanakis, Phys. Rev. B 87, 024420 (2013).

[29] I. Galanakis, P.H. Dederichs, N. Papanikolaou, Phys. Rev. B 66, 174429 (2002).

[30] E. Şaşioğlu, L.M. Sandratskii, P. Bruno, Phys. Rev. $B$ 72, 184415 (2005). 